\title{
1 Geometric correction factor for transepithelial electrical \\ 2 resistance measurements in Transwell and microfluidic cell \\ 3 cultures
}

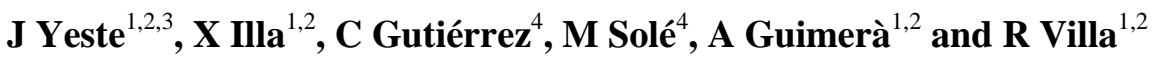

${ }^{1}$ Institut de Microelectrònica de Barcelona, IMB-CNM (CSIC). 08193, Bellaterra, Barcelona, Spain.

${ }^{2}$ CIBER-BBN, Networking Centre on Bioengineering, Biomaterials and Nanomedicine, Barcelona, Spain.

${ }^{3}$ Departamento de Microelectrónica y Sistemas Electrónicos, Universitat Autònoma de Barcelona, 08193, Bellaterra, Spain.

${ }^{4}$ Institut de Neurociències i Departament de Bioquímica i Biologia Molecular, Facultat de Medicina, Universitat Autònoma de Barcelona, Barcelona, Spain.

E-mail: jose.yeste@ csic.es

\begin{abstract}
Transepithelial electrical resistance (TEER) measurements are regularly used in in vitro models to quantitatively evaluate the cell barrier function. Although it would be expected that TEER values obtained with the same cell type and experimental setup were comparable, values reported in the literature show a large dispersion for unclear reasons. This work highlights a possible error in a widely used formula to calculate the TEER, in which it may be erroneously assumed that the entire cell culture area contributes equally to the measurement. In this study, we have numerically calculated this error in some cell cultures previously reported. In particular, we evidence that some TEER measurements resulted in errors when measuring low TEERs, especially when using Transwell inserts $12 \mathrm{~mm}$ in diameter or microfluidic systems that have small chamber heights. To correct this error, we propose the use of a geometric correction factor (GCF) for calculating the TEER. In addition, we describe a simple method to determine the GCF of a particular measurement system, so that it can be applied retrospectively. We have also experimentally validated an interdigitated electrodes (IDE) configuration where the entire cell culture area contributes equally to the measurement, and it also implements minimal electrode coverage so that the cells can be visualized alongside TEER analysis.
\end{abstract}


1 Keywords: transepithelial electrical resistance, electrical impedance spectroscopy, interdigitated electrodes, microfluidics, Transwell, geometric correction factor

\section{Introduction}

Epithelial and endothelial cells play an important role in the barrier function of many organs. These cells are arranged in continuous layers and create physical barriers to the transport of certain substances. In vitro models have proven useful in mimicking the cell barrier function present in many tissues [1-12]. Most current models are based on Transwell inserts [10-13] and microfluidic cell cultures [2-8]. Transendothelial or transepithelial electrical resistance (TEER) measurements have been consistently used in in vitro systems [11-18] to evaluate the cell barrier function, which is essential to ensure model integrity during experiments. This is an alternative technique to immunocytochemical staining and transport studies, with the advantages of being non-destructive, label-free and easily applicable in real-time. It would be expected that the TEER values obtained with the same cell type and experimental setup be comparable. However, there are discrepancies between TEER values reported in the literature [19]. Therefore, it is important to assess current measurement methods to clarify whether errors in the measurement could account for the differences between TEER values.

TEER can be obtained measuring the resistance across the cell layer at a single frequency between 0 to $100 \mathrm{~Hz}$ or using electrical impedance spectroscopy (EIS) [18,20,21]. The latter, in addition to the TEER, provides information about the capacitance of the cell layer. Since at low frequencies most of the current flows through paracellular (between cells) pathways due to the capacitance of the cell membranes [22], TEER values are closely linked with the ion transport across the paracellular space. Thus, TEER measurement allows to quantify intracellular junctions.

The electrode configurations broadly used to measure TEER are bipolar (two-electrodes) and tetrapolar (four-electrodes) [23,24] configurations. Bipolar electrode systems are commonly used [25,26] because they can be easily integrated into in vitro models, but have the disadvantage of being influenced by the electrode polarization impedance at the electrode-electrolyte interface. Tetrapolar electrode systems overcome this limitation. In these systems, the current is injected through a pair of electrodes (current carrying electrodes) while another pair of electrodes (pick-up electrodes) measures the voltage drop. Nevertheless, special care has to be taken when designing tetrapolar systems because they are more vulnerable to error than bipolar systems [27]. Moreover, small electrodes and channels in microfluidic systems imply an increase of the electrode polarization impedance, and a non-uniform electrical current distribution caused by the high electrical resistance along the microchannels.

To compare data from different studies, TEER is usually calculated by multiplying the measured resistance by the total cell culture area, resulting in a value independent of the area. This work highlights a possible error in the application of this formula, in which it may be erroneously assumed that the entire area contributes equally to the measurement. Instead of using the total cell culture area, we suggest to use an area 
to the measurement depends highly on the current distribution. Since the current distribution is influenced by the electrode configuration, the geometry of the cell culture, and even the TEER, the values reported in the literature may be scattered owing to many different measurement systems in use, especially for microfluidic cell cultures. To correct this issue, we propose the use of a geometric correction factor (GCF) for calculating the TEER.

This work presents a numerical and an experimental study of different tetrapolar electrode configurations that are currently being used for TEER measurements in Transwell and microfluidic cell cultures. We have compared the so-called 'chopstick' electrodes [29-32], the gold standard for measuring TEER in Transwell cultures, two electrode configurations integrated in microfluidic systems based on previously reported literature $[2,3,6]$ and an electrode configuration based on interdigitated electrodes (IDE) reported by our group [33]. In particular, we have used the finite element method (FEM) to study the sensitivity distribution along the cell culture area, and the GCF that should be used instead of the total cell culture area to calculate the TEER.

Herein, we show that some TEER measurements performed with the Transwell culture inserts, $12 \mathrm{~mm}$ in diameter, or the microfluidic systems that have small chamber heights lead to errors when measuring low TEERs if no GCF is used. Hence, part of the reported dispersion of TEER values could be attributed to a calculation error.

Finally, we have integrated the numerically studied IDE configuration where the entire cell culture area contributes equally to the measurement into a custom-made bioreactor to perform TEER measurements. A cell barrier model consisting of a monolayer of human cerebral microvascular endothelial cells was built in the bioreactor to experimentally validate the measurement system. In addition, this system based on IDE configuration implemented minimal electrode coverage so that the cells can be visualized alongside TEER analysis.

\section{Materials and methods}

\subsection{Simulation model}

The electrical current distribution for the different electrode configurations were analysed using FEM models developed with a commercial software package (COMSOL Multiphysics version 5.0 and its AC/DC module). Two different simulation models were developed: (1) the Transwell model (figure 1(a)) with a tetrahedral mesh of $\sim 800 \cdot 10^{3}$ elements, and (2) the microfluidic model (figure 1(b)) with a triangular mesh of $\sim 17 \cdot 10^{3}$ elements. In both models, a constant DC current of $1 \mathrm{~A}$ was injected through the current carrying electrodes, while pick-up electrodes were set as floating potential and zero current. The rest of the outer boundaries were defined as electrical insulations.

In detail, the Transwell model (model A) is a 3D representation of a conventional cell culture insert with chopstick electrodes. A cross-sectional schematic is shown in figure 1(a). The model was created for 6.5 and $12 \mathrm{~mm}$ diameter inserts. These diameters are recommended for normalizing TEER measurements performed with chopstick electrodes, and are widely used [34-40]. 


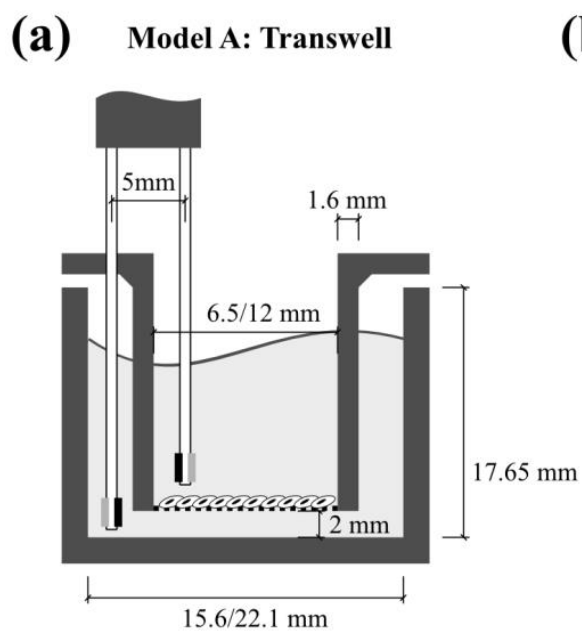

(b)

Model B

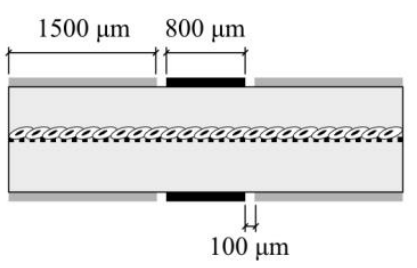

Model D: Interdigitated electrodes

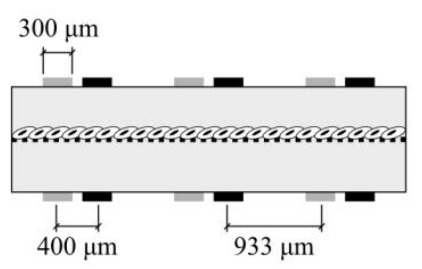

Model C
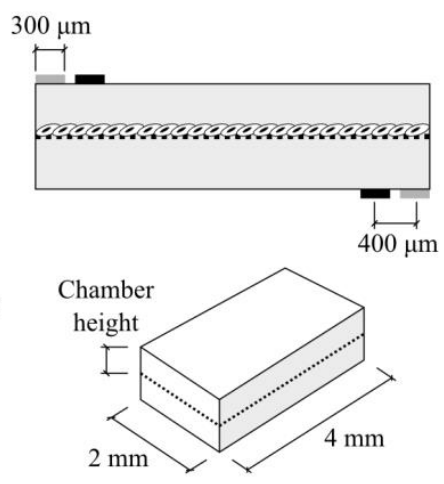

Pick-up electrodes

Current carrying electrodes

Figure 1. Geometrical dimensions for the simulated Transwell model (6.5 and $12 \mathrm{~mm}$ diameter inserts) (a), and the microfluidic model (b) with electrode configurations reported in the literature (model B-D). Chamber height is from 100 to $500 \mu \mathrm{m}$. Note that figure is not drawn to scale.

The microfluidic model (model B-D) is a 2D vertical cross-section of a microfluidic cell culture. Schemes of the different electrode configurations are shown in figure 1(b). The model comprises two superimposed microfluidic chambers, TEER measurement electrodes placed in upper and lower surfaces of the top and bottom chamber, respectively, and a small volume that represents a cell layer in the middle of the two chambers. Cell barriers with different TEER values were simulated by changing the conductivity of this volume.

Three different electrode configurations using the microfluidic model were simulated:

(1) Model B is inspired in the work by Booth et al [2]. It comprises small pick-up electrodes placed in the middle of the chambers and surrounded by large current carrying electrodes that cover $75 \%$ of the cell culture area.

(2) Model C has one pair of current carrying and pick-up electrodes at the surfaces of both chambers but in opposite sides. Despite the fact that the electrodes are planar and placed on the chamber surface, results are similar to those expected for models where wire electrodes are located at specific positions of the chamber $[6,41]$.

(3) Model D, which was proposed by the authors in Yeste et al [33], has IDE to improve the uniformity of the current distribution while reducing the space occupied by the electrodes; hence allowing microscopic visualization of the cell culture, which is usually necessary. In particular, pairs of fingers (including a pick-up and a current carrying electrode) are placed facing each other and distributed equally along the top and bottom surfaces of the top and bottom chambers, respectively.

A porous membrane that supports the cells restricts the medium path to small pores and adds a resistance in series with the cell barrier. Membranes are commercially available in a broad range of pore sizes and densities. For instance, a porous membrane of $10 \mu \mathrm{m}$ in thickness and $0.4 \mu \mathrm{m}$ of pore size will be equivalent to a resistance of $0.5 \Omega \mathrm{cm}^{2}$ and $12 \Omega \mathrm{cm}^{2}$ for pore densities of $10^{8}$ and $4 \cdot 10^{6}$ pores cm , respectively. Since TEER and transport studies require high pore density membranes to allow maximum 
1 diffusion and the resistance of these membranes are much lower than the measured resistance, we have neglected the influence of the resistance of a porous membrane.

Note that the herein presented results are obtained for a given model and geometry (figure 1), therefore, it is expected that results slightly differ for other conditions, such as different dimensions or low pore density membranes. More details about the models and simulation parameters are provided as supplementary information.

\subsection{Sensitivity evaluation}

When measuring the TEER of a cell barrier, not all the zones contribute equally to the measured impedance. Intuitively, the volumes close to the electrodes contribute more than the volumes that are far away from them. The sensitivity $s$ of each zone can be calculated as reported by Grimnes and Martinsen [27]:

$$
s=\frac{\boldsymbol{J}_{1} \boldsymbol{J}_{2}}{I^{2}}
$$

where $\boldsymbol{J}_{1}$ and $\boldsymbol{J}_{2}$ are the current density fields when a current $I$ is injected through the current carrying electrodes and pick-up electrodes, respectively. A higher absolute value of the sensitivity means a greater influence on the total measured impedance. When the electrical resistivity of a volume is increased, and if the sensitivity is positive, higher electrical impedance is measured. Otherwise, if sensitivity is negative, lower electrical impedance is measured.

\subsection{Geometric correction factor evaluation}

In Transwell and microfluidic systems, apart from the TEER, the total measured impedance also has contributions from the medium, the porous membrane support, and in case of bipolar systems, the electrode polarization impedance. For this reason, the TEER is experimentally obtained as

$$
\text { TEER }=\left(R-R_{\text {blank }}\right) A,
$$

where $R$ is the total measured impedance, $R_{\text {blank }}$ is the impedance measured without cells, and $A$ is the total cell culture area used to normalize the TEER value.

In this study, the error of the TEER measurement without GCF is calculated as the difference between the TEER obtained from simulation according to equation (2) $\left(\right.$ TEER $\left._{\mathrm{s}}\right)$, and the theoretical TEER $\left(\mathrm{TEER}_{\mathrm{t}}\right)$ used as a parameter for the electrical conductivity of the small volume in the middle of the two chambers, which represents a cell layer:

$$
\text { error }=\frac{\text { TEER }_{\mathrm{s}}-\mathrm{TEER}_{\mathrm{t}}}{\mathrm{TEER}_{\mathrm{t}}} .
$$

To correct this error and to expand the equation (2) to cases where not all the cell culture area contributes to the measurement, we propose the inclusion of a GCF in the TEER calculation:

$$
\begin{gathered}
\mathrm{GCF}=\frac{\mathrm{TEER}_{\mathrm{t}}}{\mathrm{TEER}_{\mathrm{s}}} \\
\mathrm{TEER}_{\mathrm{GCF}}=\left(R-R_{\text {blank }}\right) A \mathrm{GCF} .
\end{gathered}
$$




\subsection{Simulation strategy}

2 The numerical study was performed by applying a constant current through the current carrying electrodes (current density field $\boldsymbol{J}_{1}$ ). To calculate the sensitivity following equation (1), an additional simulation was made by exchanging pick-up and current carrying electrodes, i.e. current was applied through the previous pick-up electrodes and voltage was recorded through the previous current carrying electrodes (current density field $\boldsymbol{J}_{2}$ ). The Transwell model and each microfluidic model were simulated for different insert diameters $(6.5$ and $12 \mathrm{~mm}$ ) and different chamber heights (from 100 to $500 \mu \mathrm{m}$ ), respectively. Simulations were conducted with parametric sweeps of the $T E E R_{\mathrm{t}}$ (from 0 to $10^{3} \Omega \mathrm{cm}^{2}$ ) spanning the range of the values reported in the literature $[42,43]$. The error and GCF were calculated according to equation (3) and (4) using the current density field $J_{1}$, where $R$ and the $R_{\text {blank }}$ were obtained using Ohm's law. In particular, $R_{\text {blank }}$ was obtained when using the lowest $T E E R_{\mathrm{t}}$, as if there was no cell layer.

The simulation strategy was carried out in two steps: firstly, the sensitivity distribution along the cell barrier was simulated and compared between the different models; secondly, the error of the TEER measurement, and the GCF were calculated for each model.

\subsection{Experimental validation of IDE configuration}

An IDE configuration based on model D was evaluated using a custom-made bioreactor composed of an upper and lower plate, and a permeable membrane, where human cerebral microvascular endothelial cells were cultured to measure the TEER.

2.5.1 Bioreactor design and fabrication. Ti/Au $(20 \mathrm{~nm} / 100 \mathrm{~nm})$ electrodes were patterned on a $188 \mu \mathrm{m}$ thick cyclo-olefin polymer (COP) film (ZF14-188, Zeon Europe GmbH, DE) in a standard lift-off process. The electrodes were electrochemically coated with a layer of platinum black [44] in order to decrease the electrode polarization impedance. The COP film was bonded to a thicker COP substrate, $4 \mathrm{~mm}$ thick (Zeonor 1420R, Zeon Europe GmbH, DE), by means of a double-side pressure-sensitive adhesive (PSA) (ARcare 8939, Adhesive Research Inc., IE). Fluid inlets and outlets were defined in the plates using a CNC milling machine (MDX-40A, Roland DG Corporation, ES) and fluidic connections (male mini luer plugs, Microfluidic ChipShop GmbH, DE) were finally assembled.

A commercial porous membrane (Cyclopore polycarbonate (PC) thin clear membrane, $47 \mathrm{~mm}$ in diameter, $1 \mu \mathrm{m}$ of pore size, GE Healthcare Europe $\mathrm{GmbH}$, ES) was modified to be integrated into the bioreactor. Two PSA sheets were cut using a cutting plotter (CAMM-1 Servo GX-24, Roland DG Corporation, ES) to define the cell culture area, and bonded to both sides of the membrane. Silicone sheets (platinum cured sheet, $500 \mu \mathrm{m}$ thick, Silex Ltd., UK) were cut and placed between the plates and the membrane. Apart from defining the chamber height, the silicone sheets also act as the sealing gaskets of the system. The final assembly of the bioreactor was made by sandwiching the modified porous membrane between the upper and lower plates. More details about the bioreactor fabrication are provided as supplementary information. 
1 2.5.2 Cell culture. Human cerebral microvascular endothelial cell line hCMEC/D3 (obtained from Dr. 2 Couraud, Paris, France), which has been widely used as a model of human blood-brain barrier [45-47], was used to experimentally validate the bioreactor. The modified membranes were sterilized by exposure to UV light for $30 \mathrm{~min}$ on each side and placed on a 12-well plate. Before cell seeding, membranes were coated with collagen type $\mathrm{I}\left(150 \mu \mathrm{g} \mathrm{ml}^{-1}\right.$ in sterile distilled water), incubated at $37^{\circ} \mathrm{C}$ for $1 \mathrm{~h}$, and rinsed three times with phosphate-buffered saline (PBS). Cells were plated on the cell culture area defined on the modified membrane at a concentration of $1.8 \times 10^{5}$ cells cm${ }^{-2}$ in EBM-2 (Lonza) medium supplemented with $5 \%(\mathrm{v} / \mathrm{v})$ foetal bovine serum (FBS) (Sigma-Aldrich), 1.4 $\mu \mathrm{M}$ hydrocortisone (Sigma-Aldrich), $5 \mu \mathrm{g} \mathrm{ml}^{-1}$ ascorbic acid (Sigma-Aldrich), $1 \%$ chemically defined lipid concentrate (Life Technologies), $10 \mathrm{mM}$ HEPES (Life Technologies), $1 \mathrm{ng} \mathrm{ml}^{-1}$ human fibroblast growth factor-basic (bFGF) (Sigma-Aldrich) and the antibiotics penicillin $\left(100 \mathrm{U} \mathrm{ml}^{-1}\right)$ and streptomycin $\left(100 \mu \mathrm{g} \mathrm{ml}^{-1}\right)$. Cells were maintained at $37{ }^{\circ} \mathrm{C}$ in a humidified atmosphere containing $5 \% \mathrm{CO}_{2}$, refreshing growth medium every 2-3d until used for impedance measurements.

2.5.3 Impedance spectroscopy. TEER of the cell cultures were obtained using EIS. Impedance spectra were measured at 20 frequencies, ranging from $100 \mathrm{~Hz}$ to $1 \mathrm{MHz}$, using an impedance analysis system [48]. Impedance measurements were performed prior to cell seeding $\left(\boldsymbol{Z}_{\text {blank }}\right)$ and at $44,68,92$, and $168 \mathrm{~h}$ after cell seeding $\left(\boldsymbol{Z}_{\text {measured }}\right)$. Each membrane was used for only one measurement to minimize cell damage during handling. The $\boldsymbol{Z}_{\text {blank }}$ was then subtracted from the $\boldsymbol{Z}_{\text {measured }}$ and fitted (using the least-squares method in Matlab) to an equivalent electric circuit that describes the impedance spectra of a cell barrier. This circuit is composed of three elements: two electrical resistances that represent the paracellular (TEER) and the intracellular $\left(R_{\mathrm{i}}\right)$ pathways and a constant phase element $(\mathrm{CPE})$ that describes the dielectric properties of many cell membranes and whose impedance is

$$
\mathbf{Z}_{\mathrm{CPE}}=\frac{1}{K(\mathrm{j} \omega)^{\alpha}}
$$

where $\mathrm{j}$ is the imaginary unit, $\omega$ is the angular frequency, and $K$ and $\alpha$ are CPE coefficients. Even though the cell membrane of one cell behaves as a capacitance, a CPE provides a much better fit to the measured impedance of many cells $[49,50]$.

\section{Results and discussion}

\subsection{Sensitivity distribution}

The sensitivity distribution was calculated using the FEM models presented to determine the contribution of each zone of the cell barrier to the measured impedance. This was evaluated according to equation (1) and normalized by multiplying by the squared cell culture area. Thus, the optimal sensitivity should be constant and equal to 1 . The sensitivity profile along the cell barrier when TEER is measured in the different models is shown in figure 2. As expected, zones close to the electrodes contribute more than zones that are far away 
1 while sensitivity valleys are found in the middle of pair of electrodes, except for model B, where the major contribution comes from between the pick-up electrodes.
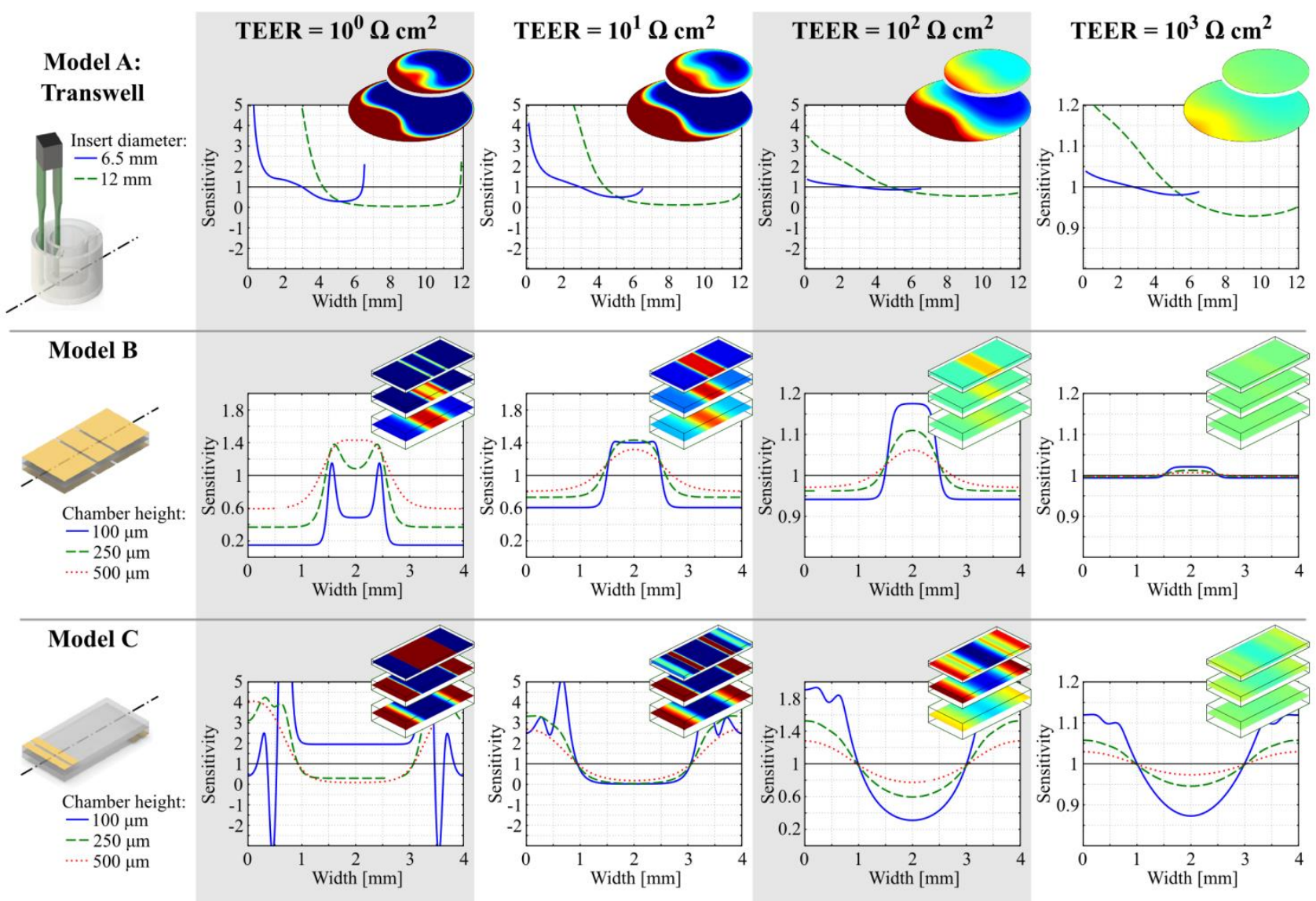

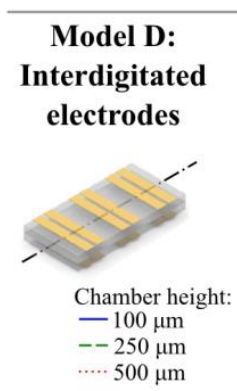

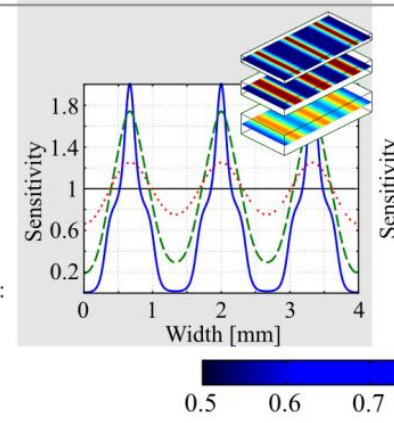
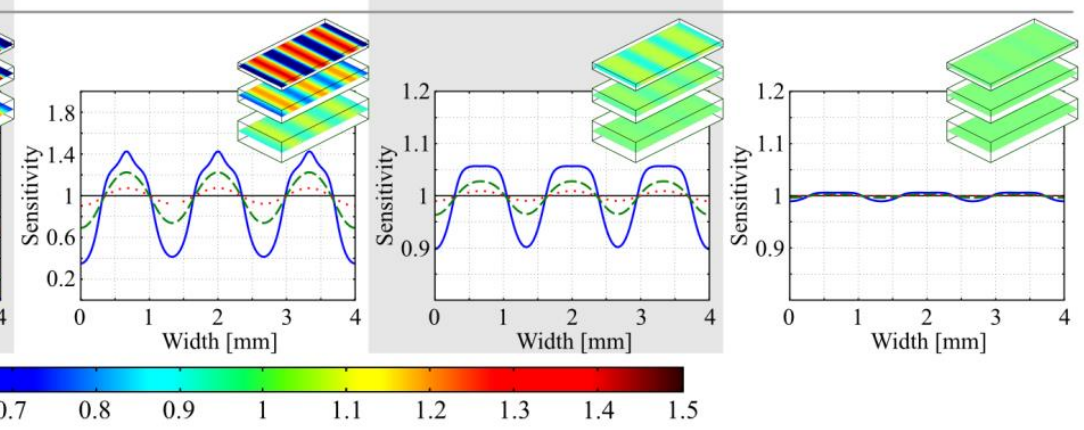

Figure 2. Sensitivity distribution along the cell barrier through the axis (dash-dotted line) shown in the 3D scheme of the model at the left of the figure when TEER is measured in a Transwell culture insert using chopstick electrodes (model A) and in the microfluidic model using the three electrode configurations (model B-D). Results are presented for different TEERs $\left(10^{0}, 10^{1}, 10^{2}, 10^{3} \Omega \mathrm{cm}^{2}\right)$, Transwell insert diameters $(6.5$ and $12 \mathrm{~mm})$ and chamber heights (100, 250 and $500 \mu \mathrm{m})$. Data are normalized by multiplying by the squared cell culture area, $A^{2}$. Attached to each line graph there is a 2D image to better clarify the sensitivity distribution. Note that axis have different scales.

In the Transwell model, the sensitivity field is highly affected by introducing the chopstick electrodes in the periphery of the Transwell insert. Consequently, the sensitivity is non-uniformly distributed and the zone close to the electrode has a higher contribution to the impedance measurement. This accounts for small variations of TEER due to non-reproducibility placement of the chopstick electrodes, which are manually positioned. For a better reproducibility of TEER measurements in Transwell inserts, some commercial 
systems automates the placement of the chopstick electrodes (REMS AutoSampler, World Precision Instruments Inc., US).

Concerning the microfluidic models, models B and D have a more uniform sensitivity than model C, which presents large differences between zones of the culture area. These differences are higher at both ends of the chamber and lower at the centre. In particular, the sensitivity at the centre is below the $25 \%$ of the optimal when measuring a TEER of $10^{1} \Omega \mathrm{cm}^{2}$. Therefore, impedance measurements performed with model $\mathrm{C}$ configuration are just representative of a small zone of the cell culture area.

A dependency of the TEER on the sensitivity has been found for all models. In particular, the higher the TEER, the more uniform is the sensitivity distribution. For the studied models, there are little sensitivity differences when TEER is greater than $10^{3} \Omega \mathrm{cm}^{2}$ (less than $5 \%$ for model B, D and Transwell inserts $6.5 \mathrm{~mm}$ in diameter, $12 \%$ for model $\mathrm{C}$, and $23 \%$ for Transwell inserts $12 \mathrm{~mm}$ in diameter).

Another feature that highly affects the sensitivity is the chamber geometry, as the sensitivity differs widely between different insert diameters and chamber heights. The sensitivity field in a Transwell insert is less uniform as its diameter increases, because larger Transwell inserts have zones further away from the chopstick electrodes that contribute little to the measurement. For example, in $12 \mathrm{~mm}$ Transwell inserts and TEERs of $10^{1} \Omega \mathrm{cm}^{2}$, the sensitivity field over half of the cell barrier area is less than $25 \%$ of the optimal sensitivity. Furthermore, decreasing the chamber height in microfluidic models is usually detrimental for the sensitivity field. Thus, the most uniform sensitivity is achieved for the larger chamber height $(500 \mu \mathrm{m})$ in all models.

\subsection{Geometric correction factor}

TEER may be calculated incorrectly using equation (2) because of a non-uniform sensitivity field. Using the equation (5) proposed by the authors, which includes a GCF, can solve this issue. The GCF value that should be used in the calculation of TEER for the different simulated models is shown in figure 3. A GCF close to 1 means that there is no error when calculating TEER using the total cell culture area. Otherwise, a GCF away from 1 means that there is a significant error.

In general, the higher the TEER, the less is the error (GCF closer to 1 as shown in figure 3). This is in agreement with the results of the previous section. In all models, GCF is closer to $1(0.95-1)$ for a TEER of $10^{3} \Omega \mathrm{cm}^{2}$ and it is expected to be maintained for higher TEERs. Unfortunately, for lower TEER values, the error considerably increases. For example, for a TEER of $10^{1} \Omega \mathrm{cm}^{2}$, GCF in Transwell inserts are 0.92 (6.5 $\mathrm{mm}$ in diameter) and 0.56 (12 $\mathrm{mm}$ in diameter), while for microfluidic models with $200 \mu \mathrm{m}$ in height are 1.32 (model B), 0.51 (model C) and 1.09 (model D). Thus, using a GCF becomes important for cell cultures with low TEERs. Another important aspect to consider is that the GCF is lower than 1 in model A and C, which means an overestimation of TEER, whereas GCF is higher than 1 in model B and D, which means an underestimation of TEER. Therefore, there will be large differences when comparing measurements systems with GCF $>1$ against those with GCF $<1$.

It is well known that the TEER measurements performed with chopstick electrodes in Transwell inserts $24 \mathrm{~mm}$ or larger in diameter should not be multiplied by their area. Nevertheless, we evidence that 
measurements of low TEER values with inserts $12 \mathrm{~mm}$ in diameter are also inaccurate and should need a GCF, as shown in figure 3(a).

For the microfluidic models, GCF value is highly dependent on the chamber height. In particular, GCF for a TEER of $10^{1} \Omega \mathrm{cm}^{2}$ varies by $27 \%$ (model B), $-44 \%$ (model C), and $12 \%$ (model D) by just changing the height from $200 \mu \mathrm{m}$ to $100 \mu \mathrm{m}$. This illustrates the importance of considering the chamber geometry and the electrode size and placement when designing TEER measurement electrodes.

(a)

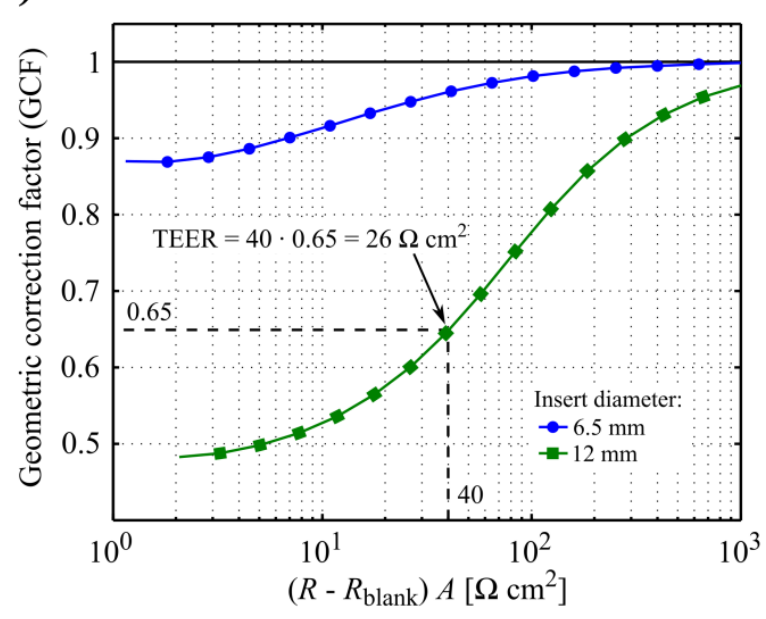

(c)

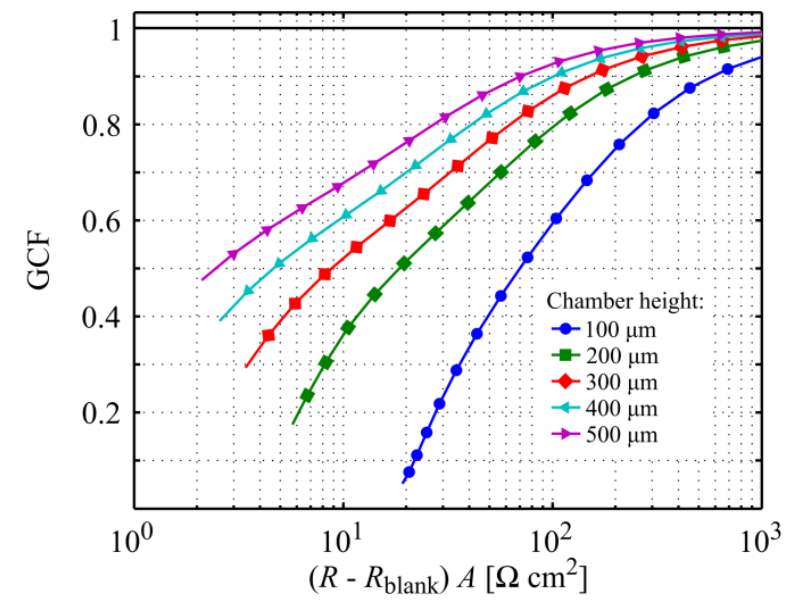

(b) Model B

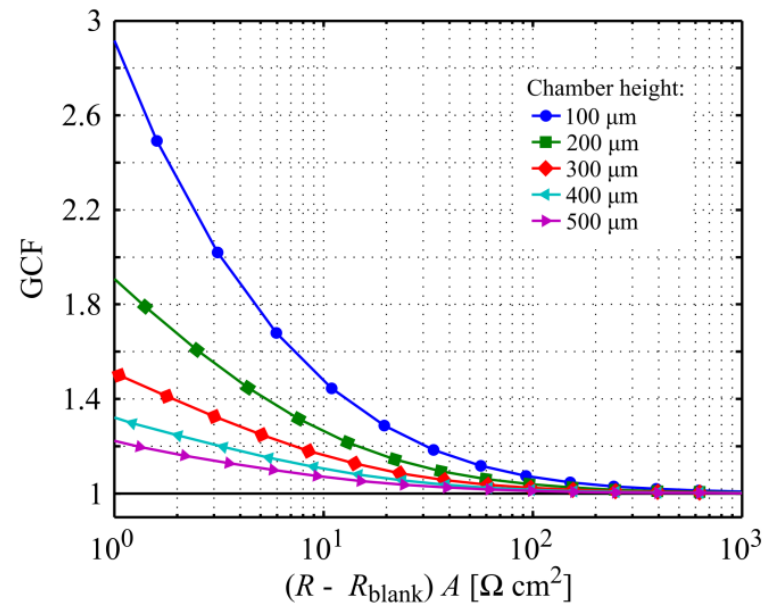

(d)

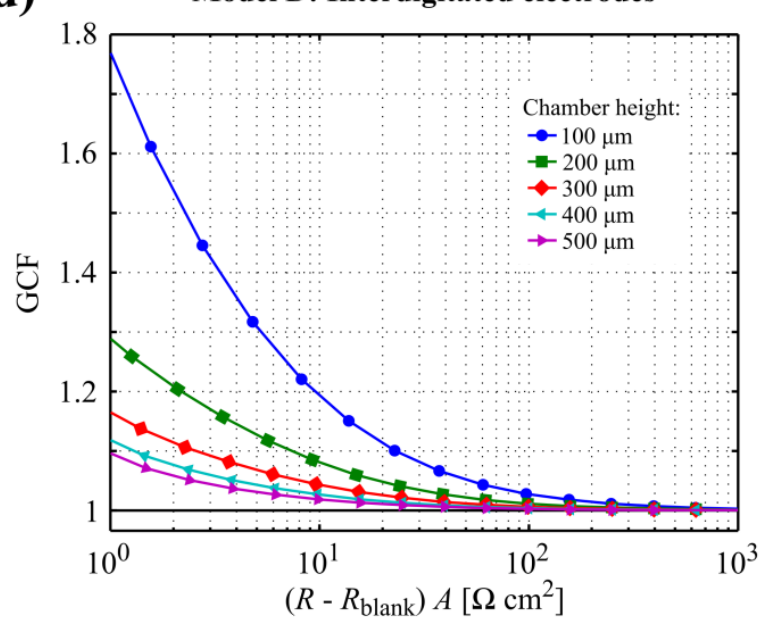

Figure 3. GCF when TEER is measured in a Transwell insert using chopstick electrodes (model A) (a) and in microfluidic models using the three electrodes configurations (models B (b), C (c) and D (d)). Results are presented for different Transwell insert diameters $(6.5$ and $12 \mathrm{~mm}$ ) and chamber heights (from 100 to $500 \mu \mathrm{m}$ ). Example of how to apply the GCF in Transwell inserts of $12 \mathrm{~mm}$ in diameter (a); a TEER of $40 \Omega \mathrm{cm}^{2}$ calculated using the total cell culture area would be $26 \Omega \mathrm{cm}^{2}$ after be corrected with the GCF.

In model $\mathrm{B}$, it could be understood that a large current electrode is more appropriate, but this is not entirely true in tetrapolar electrode configurations because there are zones of positive and negative sensitivity. In this case, maximum sensitivity is concentrated between pick-up electrodes (figure 2(b)). In model C, the accuracy is worse than in model B as the narrowness of the chamber obstruct the electrical current to flow through the cell barrier in areas away from the electrodes. This is similar to what happens in 
1 bipolar configurations with electrodes placed at inlets and outlets of the microfluidic chambers. Therefore, placing the electrodes at inlets and outlets of microfluidic systems can become a limiting factor for obtaining an accurate TEER measurement if it is determined according to equation (2) without any correction.

Interestingly, the model $\mathrm{D}$ has a $G C F$ closer to 1 than both model $\mathrm{B}$ and $\mathrm{C}$ despite covering less than half of the surface area with electrodes. An optimal accuracy in model D is achieved when the distance between the centres of the pick-up and current carrying electrodes $(400 \mu \mathrm{m})$ is comparable to the chamber height. For that reason, GCF becomes significant for chamber height of $100 \mu \mathrm{m}\left(1.2\right.$ at TEER of $\left.10^{1} \Omega \mathrm{cm}^{2}\right)$. A strategy to reduce the error in lower chamber heights could be to decrease the distance between current carrying and pick-up electrodes.

\subsection{Experimental impedance measurements}

EIS measurements performed before cell seeding and $7 \mathrm{~d}$ after seeding of an hCMEC/D3 monolayer are shown in figure 4(c). The impedance at low frequencies (associated with paracellular pathways) was purely resistive and increased from $125 \Omega$ to $359 \Omega$ after $7 \mathrm{~d}$ of cell culture, while the impedance at high frequencies (associated with paracellular and intracellular pathways) remained almost unchanged. This behaviour is due to the growth of the cell culture that implied a tightening of the space between cells.

(a)

(b)
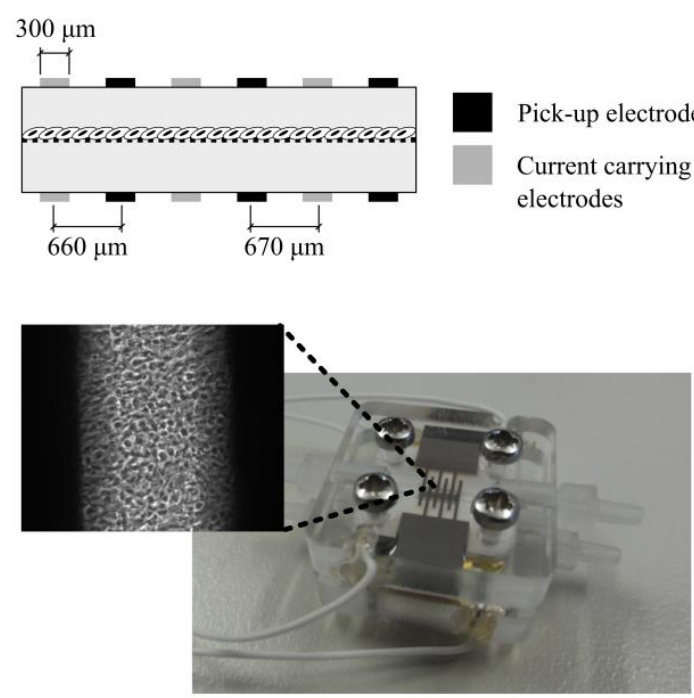

(c)
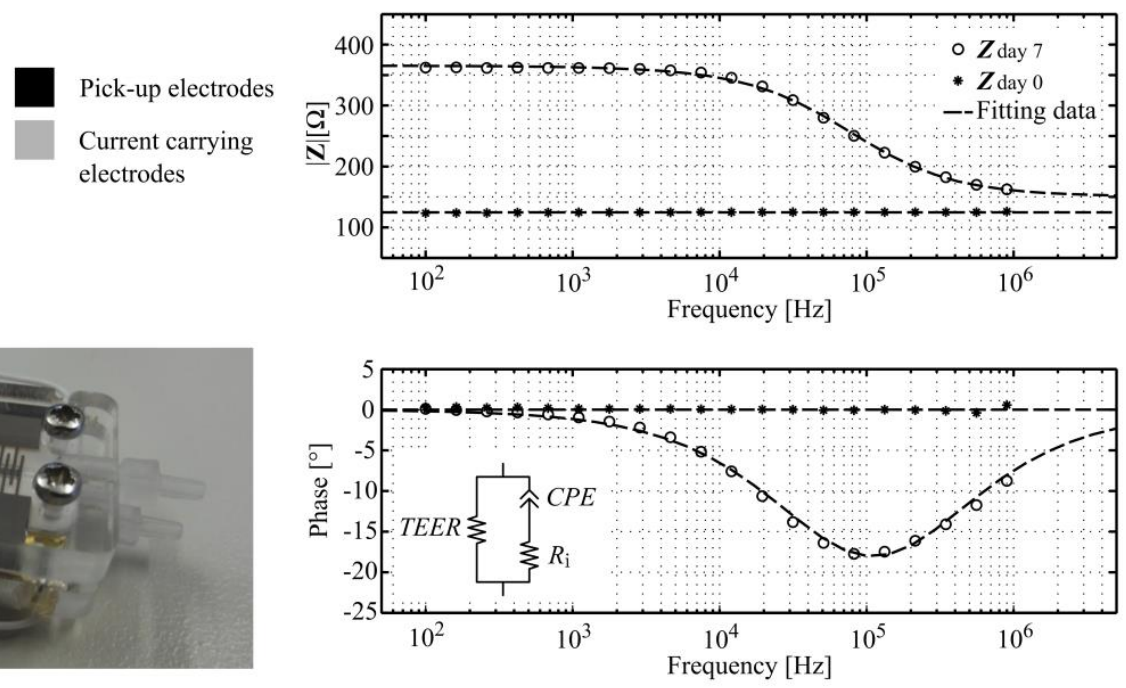

Figure 4. Bioreactor fabrication. Cross-sectional schematic of the bioreactor (a), and a picture of the bioreactor (b) with detail of cells cultured in the bioreactor. Impedance spectroscopy data (c) before cell seeding (asterisks), after $7 \mathrm{~d}$ of hCMEC/D3 culture (circles) and fitting data (dashed line) according to the equivalent electric circuit including TEER, intracellular resistance $\left(R_{\mathrm{i}}\right)$ and a CPE.

In detail, TEER increased from $10.4 \pm 1.8 \Omega \mathrm{cm}^{2}$ at $44 \mathrm{~h}$ to $18.7 \pm 2.4 \Omega \mathrm{cm}^{2}$ at $7 \mathrm{~d}$. TEER values reported in the literature for hCMEC/D3 monolayers under static conditions are typically in the range from 10 to $40 \Omega \mathrm{cm}^{2}[10,12,45]$. Concerning the $K$ parameter (related to the CPE), it decreased from 
$1 \quad 1.53 \pm 0.08 \mu \mathrm{S} \mathrm{s}^{\alpha} \mathrm{cm}^{-2}$ at $44 \mathrm{~h}$ to $1.15 \pm 0.17 \mu \mathrm{S} \mathrm{s}^{\alpha} \mathrm{cm}^{-2}$ at $7 \mathrm{~d}$. The rest of the parameters did not show any time dependency $\left(R_{\mathrm{i}}=39.6 \pm 9.8 \Omega, \alpha=0.84 \pm 0.02\right)$.

Some authors suggest that TEER could be directly determined by the resistance difference between high and low frequencies [19,28], thereby a blank measure would not be necessary. In this study, all measured impedances at $1 \mathrm{MHz}$ were not purely resistive (phase equal to $-7.1 \pm 1.9^{\circ}$ ); thus, the direct determination of TEER using the estimated resistance at high frequencies (parallel resistance of TEER and $R_{\mathrm{i}}$ ) would have implied a variation of $2.6 \pm 0.6 \Omega \mathrm{cm}^{2}$.

\section{Conclusions}

Despite the disparities of TEER values reported in the literature, few works have studied their sources. In this paper, we have analysed the importance of using a GCF to calculate the TEER, in addition to considering the sensitivity distribution when designing TEER measurement electrodes.

We have found that the errors when no GCF is used are higher for low TEER values when using larger Transwell insert diameters or lower microfluidic chamber heights. Interestingly, but not surprisingly, measuring TEER in Transwell systems using chopstick electrodes, the gold standard method in which most of the TEER values obtained in microfluidic systems are compared, is not exempt from error. We have also found that such error has different sign for different systems, resulting in large differences when comparing systems that have errors of opposite sign.

By numerical simulations, we described a simple method to determine the GCF of a particular measurement system that can correct this error, so that the correction can be applied retrospectively to their measurements. In addition, system with nonobvious current distribution that report TEER values in units of $\Omega$ instead of $\Omega \mathrm{cm}^{2}$, such as Transwell inserts of $24 \mathrm{~mm}$ in diameter or peculiar microfluidic cell cultures [51], could determine their particular GCF that allows comparing their TEER values with those reported in the literature.

We have also experimentally validated an IDE configuration based on the presented simulation results by integrating the impedance measurement electrodes in a custom-made bioreactor. This configuration, besides being more accurate for measuring the TEER, also allows the optical visualization of the cell culture. These characteristics should be very useful for the development of future microfluidic systems that pretend to emulate and monitor different cell barrier functions.

\section{Acknowledgements}

This work is part of the requirements to achieve the $\mathrm{PhD}$ degree in Electrical and Telecommunication Engineering at the Universitat Autònoma de Barcelona and it was supported by grants from CIBER-BBN, CSIC (PIE-201450E116) and Ministerio de Economía y Competitividad (SAF2014-62114-EXP and DPI2015-65401-C3-3-R). CIBER-BBN is funded by Instituto de Salud Carlos III. We are grateful to Drs. Pierre-Olivier Couraud (INSERM, France), Babette Weksler (Weill Cornell Medical College, New York, NY) and Ignacio Romero (Open University, Milton Keynes, UK) for kindly providing the hCMEC/D3 cell 
line. We would also acknowledge to Dr Mercedes Unzeta (Universitat Autònoma de Barcelona, Spain) for

\section{References}

[1] Cucullo L, Hossain M, Tierney W and Janigro D 2013 A new dynamic in vitro modular capillariesvenules modular system: Cerebrovascular physiology in a box BMC Neurosci. 1418

[2] Booth R and Kim H 2012 Characterization of a microfluidic in vitro model of the blood-brain barrier ( $\mu$ BBB) Lab. Chip 12 1784-92

[3] Walter F R, Valkai S, Kincses A, Petneházi A, Czeller T, Veszelka S, Ormos P, Deli M A and Dér A 2016 A versatile lab-on-a-chip tool for modeling biological barriers Sens. Actuators B Chem. 222 1209-19

[4] Prabhakarpandian B, Shen M-C, Nichols J B, Mills I R, Sidoryk-Wegrzynowicz M, Aschner M and Pant K 2013 SyM-BBB: a microfluidic blood brain barrier model Lab. Chip 13 1093-101

[5] Jang K-J and Suh K-Y 2010 A multi-layer microfluidic device for efficient culture and analysis of renal tubular cells Lab Chip 10 36-42

[6] Ferrell N, Desai R R, Fleischman A J, Roy S, Humes H D and Fissell W H 2010 A microfluidic bioreactor with integrated transepithelial electrical resistance (TEER) measurement electrodes for evaluation of renal epithelial cells Biotechnol. Bioeng. 107 707-716

[7] Huang H-C, Chang Y-J, Chen W-C, Harn H I-C, Tang M-J and Wu C-C 2013 Enhancement of Renal Epithelial Cell Functions through Microfluidic-Based Coculture with Adipose-Derived Stem Cells Tissue Eng. Part A 19 2024-34

[8] Huh D, Matthews B D, Mammoto A, Montoya-Zavala M, Hsin H Y and Ingber D E 2010 Reconstituting Organ-Level Lung Functions on a Chip Science 328 1662-8

[9] Illa X, Vila S, Yeste J, Peralta C, Gracia-Sancho J and Villa R 2014 A Novel Modular Bioreactor to In Vitro Study the Hepatic Sinusoid PLOS ONE 9 e111864

[10] Hatherell K, Couraud P-O, Romero I A, Weksler B and Pilkington G J 2011 Development of a threedimensional, all-human in vitro model of the blood-brain barrier using mono-, co-, and tri-cultivation Transwell models J. Neurosci. Methods 199 223-9

[11] Nakagawa S, Deli M A, Kawaguchi H, Shimizudani T, Shimono T, Kittel A, Tanaka K and Niwa M 2009 A new blood-brain barrier model using primary rat brain endothelial cells, pericytes and astrocytes Neurochem. Int. $54253-63$

[12] Eigenmann D E, Xue G, Kim K S, Moses A V, Hamburger M and Oufir M 2013 Comparative study of four immortalized human brain capillary endothelial cell lines, hCMEC/D3, hBMEC, TY10, and BB19, and optimization of culture conditions, for an in vitro blood-brain barrier model for drug permeability studies Fluids Barriers CNS 1033

[13] Tsata V, Velegraki A, Ioannidis A, Poulopoulou C, Bagos P, Magana M and Chatzipanagiotou S 2016 Effects of Yeast and Bacterial Commensals and Pathogens of the Female Genital Tract on the Transepithelial Electrical Resistance of HeLa Cells Open Microbiol. J. 10 90-6

[14] Wegener J, Abrams D, Willenbrink W, Galla H-J and Janshoff A 2004 Automated multi-well device to measure transepithelial electrical resistances under physiological conditions BioTechniques 37590 7 
[15] Gaillard P J, Voorwinden L H, Nielsen J L, Ivanov A, Atsumi R, Engman H, Ringbom C, De Boer A $\mathrm{G}$ and Breimer D D 2000 Establishment and functional characterization of an in vitro model of the blood-brain barrier, comprising a co-culture of brain capillary endothelial cells and astrocytes Eur. J. Pharm. Sci. 12 215-22

[16] Benson K, Cramer S and Galla H-J 2013 Impedance-based cell monitoring: barrier properties and beyond Fluids Barriers CNS 105

[17] Vogel P A, Halpin S T, Martin R S and Spence D M 2011 Microfluidic transendothelial electrical resistance measurement device that enables blood flow and postgrowth experiments Anal. Chem. 83 4296-301

[18] Srinivasan B, Kolli A R, Esch M B, Abaci H E, Shuler M L and Hickman J J 2015 TEER Measurement Techniques for In Vitro Barrier Model Systems J. Lab. Autom. 20 107-26

[19] Odijk M, Meer A D van der, Levner D, Kim H J, Helm M W van der, Segerink L I, Frimat J-P, Hamilton G A, Ingber D E and Berg A van den 2015 Measuring direct current trans-epithelial electrical resistance in organ-on-a-chip microsystems Lab. Chip 15 745-52

[20] Sun T, Swindle E J, Collins J E, Holloway J A, Davies D E and Morgan H 2010 On-chip epithelial barrier function assays using electrical impedance spectroscopy Lab. Chip 10 1611-7

[21] Sarró E, Lecina M, Fontova A, Gòdia F, Bragós R and Cairó J J 2016 Real-time and on-line monitoring of morphological cell parameters using electrical impedance spectroscopy measurements: Use of EIS for cell morphology monitoring J. Chem. Technol. Biotechnol. 91 1755-62

[22] Schwan H P and Kay C F 1957 The Conductivity of Living Tissues Ann. N. Y. Acad. Sci. 65 1007-13

[23] Bera T K 2014 Bioelectrical Impedance Methods for Noninvasive Health Monitoring: A Review $J$. Med. Eng. 2014 1-28

[24] Brown B H, Wilson A J and Bertemes-Filho P 2000 Bipolar and tetrapolar transfer impedance measurements from volume conductor Electron. Lett. 36 2060-2

[25] Griep L M, Wolbers F, Wagenaar B de, Braak P M ter, Weksler B B, Romero I A, Couraud P O, Vermes I, Meer A D van der and Berg A van den 2013 BBB ON CHIP: microfluidic platform to mechanically and biochemically modulate blood-brain barrier function Biomed. Microdevices 15145 50

[26] Douville N J, Tung Y-C, Li R, Wang J D, El-Sayed M E H and Takayama S 2010 Fabrication of twolayered channel system with embedded electrodes to measure resistance across epithelial and endothelial barriers Anal. Chem. 82 2505-11

[27] Grimnes S and Martinsen Ø G 2007 Sources of error in tetrapolar impedance measurements on biomaterials and other ionic conductors J. Phys. Appl. Phys. 40 9-14

[28] Wegener J and Seebach J 2014 Experimental tools to monitor the dynamics of endothelial barrier function: a survey of in vitro approaches Cell Tissue Res. 355 485-514

[29] Blume L-F, Denker M, Gieseler F and Kunze T 2010 Temperature corrected transepithelial electrical resistance (TEER) measurement to quantify rapid changes in paracellular permeability Pharmazie $\mathbf{6 5}$ $19-24$

[30] Smith J, Wood E and Dornish M 2004 Effect of Chitosan on Epithelial Cell Tight Junctions Pharm. Res. 21 43-9

[31] Gye M C 2003 Changes in the expression of claudins and transepithelial electrical resistance of mouse Sertoli cells by Leydig cell coculture Int. J. Androl. 26 271-8 
[32] Fajdiga S, Koninkx J F J G, Tooten P C J and Marinšek-Logar R 2006 Interference of Salmonella enteritidis and Lactobacillus spp. with IL-8 levels and transepithelial electrical resistance of enterocytelike caco-2 cells Folia Microbiol. (Praha) 51 268-72

[33] Yeste $\mathrm{J}$, Illa X, Guimerà $\mathrm{A}$ and Villa R 2015 A novel strategy to monitor microfluidic in-vitro bloodbrain barrier models using impedance spectroscopy Proc. SPIE 9518, Bio-MEMS and Medical Microdevices II (Barcelona, ES, 4-6 May 2015) ed S van den Driesche (Bellingham, WA: SPIE) p $95180 \mathrm{~N}$

[34] Neuhaus W, Plattner V E, Wirth M, Germann B, Lachmann B, Gabor F and Noe C R 2008 Validation of in vitro cell culture models of the blood-brain barrier: Tightness characterization of two promising cell lines J. Pharm. Sci. 97 5158-75

[35] Sultana R, McBain A J and O’Neill C A 2013 Strain-Dependent Augmentation of Tight-Junction Barrier Function in Human Primary Epidermal Keratinocytes by Lactobacillus and Bifidobacterium Lysates Appl. Environ. Microbiol. 79 4887-94

[36] Xie Y, Ye L, Zhang X, Cui W, Lou J, Nagai T and Hou X 2005 Transport of nerve growth factor encapsulated into liposomes across the blood-brain barrier: In vitro and in vivo studies $J$. Controlled Release 105 106-19

[37] Man S, Ubogu E E, Williams K A, Tucky B, Callahan M K and Ransohoff R M 2008 Human Brain Microvascular Endothelial Cells and Umbilical Vein Endothelial Cells Differentially Facilitate Leukocyte Recruitment and Utilize Chemokines for T Cell Migration J. Immunol. Res. 2008 1-8

[38] Calabro A R, Konsoula R and Barile F A 2008 Evaluation of in vitro cytotoxicity and paracellular permeability of intact monolayers with mouse embryonic stem cells Toxicol. In Vitro 22 1273-84

[39] Bridger P S, Menge C, Leiser R, Tinneberg H-R and Pfarrer C D 2007 Bovine Caruncular Epithelial Cell Line (BCEC-1) Isolated from the Placenta Forms a Functional Epithelial Barrier in a Polarised Cell Culture Model Placenta 28 1110-7

[40] Ablonczy Z and Crosson C E 2007 VEGF modulation of retinal pigment epithelium resistance Exp. Eye Res. 85 762-71

[41] Huang C, Ramadan Q, Wacker J B, Tekin H C, Ruffert C, Vergères G, Silacci P and Gijs M a. M 2014 Microfluidic chip for monitoring $\mathrm{Ca} 2+$ transport through a confluent layer of intestinal cells $R S C A d v$. 4 52887-91

[42] Garberg P et al 2005 In vitro models for the blood-brain barrier Toxicol. In Vitro 19 299-334

[43] Deli M A, Ábrahám C S, Kataoka Y and Niwa M 2005 Permeability Studies on In Vitro Blood-Brain Barrier Models: Physiology, Pathology, and Pharmacology Cell. Mol. Neurobiol. 25 59-127

[44] Gabriel G, Erill I, Caro J, Gómez R, Riera D, Villa R and Godignon P 2007 Manufacturing and full characterization of silicon carbide-based multi-sensor micro-probes for biomedical applications Microelectron. J. 38 406-15

[45] Weksler B B et al 2005 Blood-brain barrier-specific properties of a human adult brain endothelial cell line FASEB J. 19 1872-4

[46] Poller B, Gutmann H, Krähenbühl S, Weksler B, Romero I, Couraud P-O, Tuffin G, Drewe J and Huwyler J 2008 The human brain endothelial cell line hCMEC/D3 as a human blood-brain barrier model for drug transport studies J. Neurochem. 107 1358-68

[47] Cucullo L, Couraud P-O, Weksler B, Romero I-A, Hossain M, Rapp E and Janigro D 2008 Immortalized human brain endothelial cells and flow-based vascular modeling: A marriage of convenience for rational neurovascular studies J. Cereb. Blood Flow Metab. 28 312-28 
[48] Guimerà A, Gabriel G, Parramon D, Calderón E and Villa R 2009 Portable 4 Wire Bioimpedance Meter with Bluetooth Link World Congress on Medical Physics and Biomedical Engineering (Munich, DE, 7-12 September 2009) vol 25/7, ed O Dössel et al (Berlin: Springer) pp 868-71

[49] Grimnes S and Martinsen Ø G 2008 Bioimpedance and Bioelectricity Basics, 2nd Edition (Amsterdam: Elsevier) pp 283-332

[50] Ando Y, Mizutani K and Wakatsuki N 2014 Electrical impedance analysis of potato tissues during drying J. Food Eng. 121 24-31

[51] Deosarkar S P, Prabhakarpandian B, Wang B, Sheffield J B, Krynska B and Kiani M F 2015 A Novel Dynamic Neonatal Blood-Brain Barrier on a Chip PLOS ONE 10 e0142725 